\title{
Impurity induced Dirac point smearing in graphene
}

\author{
Yuriy V. Skrypnyk \\ Kurdyumov Institute of Metal Physics, 36 Vernadsky Ave., Kyiv 03680, Ukraine \\ E-mail: skrypnyk@i.com.ua \\ Vadim M. Loktev \\ Bogolyubov Institute for Theoretical Physics, 14-b Metrolohichna Str., Kyiv 03680, Ukraine \\ Received January 25, 2007
}

\begin{abstract}
It is shown that in a two-dimensional system with the linear dispersion a resonance is present in the Dirac point vicinity, when the impurity perturbation magnitude exceeds the bandwidth. The corresponding spectrum rearrangement, which follows at a certain critical impurity concentration, results in the square root dependence of the concentration smearing region width on the concentration. If the perturbation magnitude does not exceed the bandwidth, or the critical concentration is not reached, the concentration smearing region width remains exponentially small.
\end{abstract}

PACS: 71.23.An Theories and models; localized states;

71.30.+h Metal-insulator transitions and other electronic transitions.

Keywords: graphene, Dirac spectrum, impurity, spectrum rearrangement.

\section{Introduction}

Graphene is a material that has been obtained around two years ago [1]. It looks especially fresh when compared to other traditional subjects of investigation in solid state physics. New theoretical questions connected with this material immediately formed a nucleus for the area of active research. Intriguing properties of graphene are calling upon their prompt explanation not only because of the pure scientific interest, but also because of the highly possible forthcoming practical implementation of this material.

It is well known that the localizing action of impurity centers is, generally speaking, more pronounced in lowdimensional systems. However, a number of experiments evidently demonstrate that graphene, which is the first truly two-dimensional system, is highly tolerant to impurity effects. Basically, the effect of impurities on elementary excitation spectra in disordered systems is determined by the quotient of the system's dimensionality and the exponent in its dispersion relation. When this quotient is larger than one and half, the system possesses an increased dimensionality, and it behaves like a low-dimensional when the quotient is less than unity [2,3]. Because of the unusual linear electron dispersion close to the Fermi level, graphene should manifest some properties that are characteristic to four-dimensional systems, which is favorable for the localization reduction. Based on the proven method of cluster expansions, we suggest possible clues for the unique robustness of graphene to the impurity induced perturbation and describe the process of the spectrum rearrangement, which occurs on increasing the impurity concentration.

The spectrum rearrangement has been studied for a large variety of disordered systems [4-6]. The physical reason of this phenomenon roots in the spatial overlap of the impurity states that have large effective radii. The impurity state radius can far exceed the lattice constant, when its energy is close to any of the van Hove singularities in the spectrum. This overlap results in a qualitative change in the dispersion of carriers and the density of states of the disordered system. Our study is dedicated to the 90th anniversary of I.M. Lifshitz, whose contribution to the physics of disordered systems can not be overemphasized.

\section{Model}

A model for the host should be simple enough in order to make a certain advance in analytical calculations, and, at the same time, it should retain all the physics of the electron subsystem in graphene that is essential for the 
impurity problem. It has been confirmed by ARPES $[7,8]$ that the electron dispersion in graphene can be quite successfully reproduced within the tight-binding scheme by taking into account only the nearest neighbors hopping. As it follows from this approach, the total electron density of states (DOS) $\rho(\varepsilon)$ in the pure graphene sheet depends linearly on energy $\varepsilon$ in the vicinity of the Dirac point, i.e., $\rho(\varepsilon) \sim|\varepsilon|$. This result is indirectly supported by the unconventional quantum Hall effect in graphene $[9,10]$ and has been verified by strait measurements [7]. It will be assumed below that this linear behavior of the DOS is maintained for the whole energy range inside the adjacent bands, which preserves their actual symmetry in the unperturbed system. In addition, because of the symmetry of the underlying honeycomb graphene lattice, diagonal elements of the Green's function (GF) must be identical on both sublattices, $g_{\mathbf{n} 1 \mathbf{n} 1}(\varepsilon)=g_{\mathbf{n} 2 \mathbf{n} 2}(\varepsilon)$, where numbers denote sublattices, and $\mathbf{n}$ ranges over lattice cells. Since $\rho(\varepsilon)$ is the sum of the DOSs for the corresponding sublattices $\rho_{\alpha}(\varepsilon)$, it should be demanded that

$$
\rho_{\alpha}(\varepsilon)=-\frac{1}{\pi} \operatorname{Im} g_{\mathbf{n} \alpha \mathbf{n} \alpha}(\varepsilon)=\xi|\varepsilon|, \quad \rho(\varepsilon)=\sum_{\alpha=1}^{2} \rho_{\alpha}(\varepsilon),
$$

where $\xi$ is some constant. The normalization condition for the conduction band implies that

$$
\int_{0}^{W} \sum_{\alpha=1}^{2} \rho_{\alpha}(\varepsilon) d \varepsilon=1,
$$

where $W$ is the bandwidth. The same holds true for the valence band as well. These relations define the value of the constant $\xi$,

$$
\int_{-W}^{0} \rho_{\alpha}(\varepsilon) d \varepsilon=\int_{0}^{W} \rho_{\alpha}(\varepsilon) d \varepsilon=\xi \frac{W^{2}}{2}=\frac{1}{2}, \rho_{\alpha}(\varepsilon)=\frac{|\varepsilon|}{W^{2}} .
$$

In a turn, real parts of the diagonal elements of the host GF must comply with the Kramers-Kronig relation

$$
\begin{aligned}
\operatorname{Re} g_{\mathbf{n} \alpha \mathbf{n} \alpha}(\varepsilon) & =\frac{1}{W^{2}}\left(\int_{0}^{W} \frac{\varepsilon^{\prime}}{\varepsilon-\varepsilon^{\prime}} d \varepsilon^{\prime}+\int_{-W}^{0}-\frac{\varepsilon^{\prime}}{\varepsilon-\varepsilon^{\prime}} d \varepsilon^{\prime}\right)= \\
& =\frac{\varepsilon}{W^{2}} \ln \frac{\varepsilon^{2}}{\varepsilon^{2}-W^{2}} .
\end{aligned}
$$

Combining the obtained results, finally one has

$$
g_{\mathbf{n} \alpha \mathbf{n} \alpha}(\varepsilon)=\frac{\varepsilon}{W^{2}}\left[\ln \frac{\varepsilon^{2}}{W^{2}-\varepsilon^{2}}-i \pi \operatorname{sign}(\varepsilon)\right] .
$$

The introduced magnitude $W$ should be brought into correspondence, at least qualitatively, with the factual bandwidth in graphene. It had been mentioned already that the graphene Hamiltonian with the interaction between nearest neighbors $t$ yields a reasonable approximation for the electron dispersion law. According to it, in the close vicinity of the Dirac point

$$
\varepsilon(\mathbf{k}) \approx \pm v_{F} k, \quad v_{F}=\frac{\sqrt{3} a t}{2}, \quad k a<<1, \quad \hbar=1,
$$

where the vertex of the Dirac cone is taken as the reference point for the wave vector $\mathbf{k}$, and $a$ is the lattice constant [11]. Because there are two nonequivalent Dirac cones in the Brillouin zone of graphene, the corresponding tight-binding DOS is given by

$\rho_{t-b}(\varepsilon) \approx 2\left(\frac{\sqrt{3} a^{2}}{4 \pi}\right) \int d k k \delta\left(|\varepsilon|-v_{F} k\right)=\frac{2|\varepsilon|}{\sqrt{3} \pi t^{2}}, \quad|\varepsilon|<<3 t$.

The total DOS in the devised model should match at the low energies with the latter expression. Both DOSs agree with each other, when

$$
W=\sqrt{\sqrt{3} \pi} t .
$$

Thus, the bandwidth in the model with the triangular DOS is noticeably less than the graphene bandwidth, which is equal to $3 t$ within the tight-binding approximation. This result should be expected, since the triangular DOS manifests the unnatural rise towards the band edges. However, our analysis will be focused only on the effects at the low energies, where this model is fully adequate. So, the meaningful expression for the diagonal elements of the GF in the host reads (cp. (5))

$$
g_{\mathbf{n} \alpha \mathbf{n} \alpha}(\varepsilon) \approx \frac{\varepsilon}{W^{2}}\left[\ln \left(\frac{\varepsilon^{2}}{W^{2}}\right)-i \pi \operatorname{sign}(\varepsilon)\right], \varepsilon<<W .
$$

From here and on we let the bandwidth $W$ be unity, which specifies the energy scale.

As regards the impurity perturbation, we take for granted that it can be described within the well-known model of a substitutional binary alloy with the diagonal disorder. The origin of this model can be traced back to the pioneering works of Lifshitz [12]. According to it, impurities are supposed to be distributed absolutely at random in space. On-site potentials are allowed to attain only two different values depending on the type of the atom that is occupying the respective lattice site. In the asymmetric scheme of the impurity perturbation, the on-site potentials are $V_{L}$ with the probability $c$, which corresponds to impurities, or 0 with the probability $1-c$, which corresponds to host atoms. Thus, the full tight-binding Hamiltonian of the disordered system can be expressed as the sum of the translationally invariant host Hamiltonian $\mathbf{H}_{0}$ and the impurity induced perturbation $\mathbf{H}_{\text {imp }}$, 


$$
\mathbf{H}=\mathbf{H}_{0}+\mathbf{H}_{\text {imp }}, \mathbf{H}_{\mathrm{imp}}=V_{L} \sum_{\mathbf{n}, \alpha}^{\prime} c_{\mathbf{n} \alpha}^{\dagger} c_{\mathbf{n} \alpha},
$$

where $c_{\mathbf{n} \alpha}^{\dagger}$ and $c_{\mathbf{n} \alpha}$ are electron creation and annihilation operators at the lattice site situated on the sublattice $\alpha$ in the cell with radius-vector $\mathbf{n}$. The prime over the summation sign denotes that the summation in (10) is restricted to those sites that are occupied by impurities. The local character of the impurity perturbation justifies the choice of the simplified model with the triangular DOS for the host.

\section{Single impurity problem}

Let us consider the case of an isolated single impurity in the otherwise undistorted graphene lattice. It is not difficult to calculate the diagonal element of the GF $\mathcal{G}=(\varepsilon-\mathbf{H})^{-1}$ at the lattice site the impurity is positioned right on,

$$
\mathcal{G}_{0}(\varepsilon)=\frac{g_{0}(\varepsilon)}{1-V_{L} g_{0}(\varepsilon)}, g_{0}(\varepsilon) \equiv g_{\mathbf{n} \alpha \mathbf{n} \alpha}(\varepsilon)
$$

The corresponding expression for the local density of states (LDOS) at the impurity site can be unfolded by substituting the model host GF (9) into this relation,

$$
\rho_{\mathrm{imp}}(\varepsilon)=-\frac{1}{\pi} \operatorname{Im} \mathcal{G}_{0}(\varepsilon) \approx \frac{|\varepsilon|}{\left(1-2 V_{L} \varepsilon \ln |\varepsilon|\right)^{2}+\left(\pi V_{L} \varepsilon\right)^{2}} .
$$

It is evident, that for the sufficiently large impurity perturbation $V_{L}$ there is a marked peak in the impurity site LDOS. The energy $\varepsilon_{r}$, at which this peak is situated, is determined, as it should be, by the renowned Lifshitz equation,

$$
1=V_{L} \operatorname{Re} g_{0}\left(\varepsilon_{r}\right) \approx 2 V_{L} \varepsilon_{r} \ln \left|\varepsilon_{r}\right|
$$

The peak at $\varepsilon_{r}$ sits above the Dirac point, when the parameter $V_{L}<0$, and vice versa. This unusual positioning property holds true for arbitrary two symmetric bands, which touch each other at a certain energy. The denominator in (12) can be expanded about $\varepsilon_{r}$ :

$\rho_{\text {imp }}(\varepsilon) \approx \frac{|\varepsilon| \Gamma_{r}^{2}}{\left[\pi V_{L} \varepsilon_{r}\right]^{2}\left[\left(\varepsilon-\varepsilon_{r}\right)^{2}+\Gamma_{r}^{2}\right]}, \Gamma_{r}=\frac{\pi\left|\varepsilon_{r}\right|}{2|1+\ln | \varepsilon_{r}||}$,

when the condition

$$
\gamma_{r} \equiv \frac{\Gamma_{r}}{\left|\varepsilon_{r}\right|}=\frac{\pi}{2|1+\ln | \varepsilon_{r}||}<<1
$$

is met. The inequality (15) will be fulfilled provided the energy $\varepsilon_{r}$ is in the narrow vicinity of the Dirac point. According to (13), this corresponds to a large in the absolute value $V_{L}$. The physics of the condition (15) lies in the smallness of the effective impurity state damping $\Gamma_{r}$ com- pared to the distance from the energy $\varepsilon_{r}$ to the nearest van Hove singularity in the spectrum, which role plays the Dirac point in the present case. This means, that the state with $\varepsilon_{r}$ can be regarded as a well-defined impurity resonance, when the condition (15) holds. Simultaneously, the LDOS is getting the Lorentz shape near the resonance energy. In contrast, a well-defined resonance state cannot appear near the band edge of two- and three-dimensional single-band systems with the common quadratic dispersion of electrons within the Lifshitz model (10) for any possible combination of impurity and host parameters.

The difference $\Delta \rho(\varepsilon)$ between the total DOS of the single impurity system and the host can be also calculated analytically:

$$
\begin{aligned}
& \Delta \rho(\varepsilon)=\frac{V_{L}}{\pi N} \operatorname{Im}\left(\frac{d g_{0}(\varepsilon)}{d \varepsilon} \frac{1}{1-V_{L} g_{0}(\varepsilon)}\right)= \\
& =-\frac{V_{L} \operatorname{sign}(\varepsilon)\left(1+2 V_{L} \varepsilon\right)}{N\left[\left(1-2 V_{L} \varepsilon \ln |\varepsilon|\right)^{2}+\left(V_{L} \pi \varepsilon\right)^{2}\right]} .
\end{aligned}
$$

It is not hard to check that electron states of the host are redistributed within their respective bands. For, say, $V_{L}<0$, some amount of states is removed from the valence band to the split off local level, while in the conduction band states are pushed from its both sides towards the resonance energy.

There is a pronounced step in $\Delta \rho(\varepsilon)$ near the Dirac point, which is combined with the negative dip. Since the host DOS is zero (see (1)) at the Dirac point and is rather small in its neighborhood, the close vicinity of the Dirac point can not be properly described by a straightforward expansion of the impurity system GF in powers of the concentration, as it was done in [13], even if the impurity concentration is negligibly small. This is quite clear from the obvious fact that the width of this negative dip is of the same order as the resonance state damping $\Gamma_{r}$. Therefore, other approaches should be utilized to study impurity effects on the graphene spectrum in the vicinity of the Dirac point.

\section{Finite impurity concentration}

Only renormalized approaches, the most widely used among them is the coherent-potential approximation (CPA) [14], can be efficient close to the van Hove singularities in the spectrum of a disordered system. The renormalization is necessary not only to take into account the effective shift of the singularity position, but mainly to properly describe the nonuniform shift of adjacent states at a finite impurity concentration. Of course, same applies to the neighborhood of the Dirac point in graphene. Since the CPA does not contain an applicability criterion in itself, the method of cluster expansions $[15,16]$ will be used to go beyond the CPA and to estimate 
its validity. Averaged over impurity distributions GF of the disordered system $\mathbf{G}$ is related to the host GF by the Dyson equation

$$
\mathbf{G}=\mathbf{g}+\mathbf{g} \Sigma \mathbf{G} .
$$

Because the translational invariance is restored by the configurational averaging, the self-energy $\Sigma$ can be expanded into a series as follows:

$$
\Sigma_{\alpha \beta}(\mathbf{k})=\delta_{\alpha \beta} \sigma^{(1)}+\sigma_{\alpha \beta}^{(2)}(\mathbf{k})+\ldots,
$$

where the momentum-independent $\sigma^{(1)}$ is the CPA self-energy,

$$
\sigma^{(1)}=\frac{c V_{L}}{1-\left(V_{L}-\sigma^{(1)}\right) g_{0}\left(\varepsilon-\sigma^{(1)}\right)},
$$

which is to be determined self-consistently, and $\sigma_{\alpha \beta}^{(2)}(\mathbf{k})$ represents the contribution from scatterings on all possible pair impurity clusters:

$$
\begin{aligned}
& \sigma_{\alpha \beta}^{(2)}(\mathbf{k})=\sum_{l, m} \xi_{l} \xi_{m}\left(\delta_{\alpha \beta} \sum_{\mathbf{n} \gamma \neq 0 \alpha} \frac{\tau_{l}^{3} \tau_{m}^{2} \mathbf{G}_{0 \alpha \mathbf{n} \gamma}^{2} \mathbf{G}_{\mathbf{n} \gamma 0 \alpha}^{2}}{1-\tau_{l} \tau_{m} \mathbf{G}_{0 \alpha \mathbf{n} \gamma} \mathbf{G}_{\mathbf{n} \gamma 0 \alpha}}+\right. \\
& \left.+\sum_{\mathbf{n} \beta \neq 0 \alpha} \frac{\tau_{l}^{2} \tau_{m}^{2} \mathbf{G}_{0 \alpha \mathbf{n} \beta}^{2} \mathbf{G}_{\mathbf{n} \beta 0 \alpha}}{1-\tau_{l} \tau_{m} \mathbf{G}_{0 \alpha \mathbf{n} \beta} \mathbf{G}_{\mathbf{n} \beta 0 \alpha}} \mathrm{e}^{i \mathbf{k n}}\right)
\end{aligned}
$$

Here the single-site T-matrix is denoted by

$$
\tau_{l}=\frac{V_{l}-\sigma^{(1)}}{1-\left(V_{l}-\sigma^{(1)}\right) g_{0}\left(\varepsilon-\sigma^{(1)}\right)},
$$

and indices $l$ and $m$ enumerate two atom types of the binary alloy. According to this index, the factor $\xi_{l}$ attains the value $c$ for the impurities or $1-c$ for the host atoms, while the variable $V_{l}$ is $V_{L}$ or 0 , respectively.

Thorough analysis of the series for the self-energy indicates that this expansion indeed has the small parameter. As a rough guide, the self-energy series can be considered as a geometric progression with the common ratio

$$
R(\varepsilon)=\sum_{l} \xi_{l} \tau_{l}^{2} \sum_{\mathbf{n} \beta \neq 0 \alpha} \mathbf{G}_{0 \alpha \mathbf{n} \beta}^{2},
$$

and the scale factor $\sigma^{(1)}$. On approaching any van Hove singularity, the contribution from cluster scattering is increasing in magnitude. As follows from the above treatment, terms corresponding to the cluster scattering can be omitted if their sum does not exceed by the absolute value the CPA self-energy $\sigma^{(1)}$ [17],

$$
|R(\varepsilon)| \leq 1 / 2 .
$$

This inequality outlines those energy domains, where the CPA is applicable. If the criterion (23) is fulfilled, only the first single-site term can be retained in the series. Moreover, at the low impurity concentration, the multiple-occupancy corrections that are incorporated into the CPA expression can be also neglected. Thus, the so-called method of the modified propagator can be employed (cp. (19)),

$$
\Sigma \approx \sigma \mathbf{I}, \quad \sigma=\frac{c V_{L}}{1-V_{L} g_{0}(\varepsilon-\sigma)}, \quad c<<1 .
$$

Because just the close vicinity of the Dirac point is examined, the diagonal element of the host GF can be taken in the form (9). Then, by means of the conventional substitution $\varepsilon-\sigma=\kappa \exp (i \varphi), 0<\varphi<\pi$, the imaginary part of (24) becomes

$$
\begin{aligned}
& c V_{L}^{2}[2 \ln \kappa+(2 \varphi-\pi) \cot \varphi]+ \\
& +\left[1-V_{L} \kappa(2 \ln \kappa \cos \varphi-(2 \varphi-\pi) \sin \varphi)\right]^{2}+ \\
& +\left[V_{L} \kappa(2 \ln \kappa \sin \varphi+(2 \varphi-\pi) \cos \varphi)\right]^{2}=0 .
\end{aligned}
$$

If (25) is considered as an equation in the unknown $\varphi$, it always has two solutions for each chosen modulus $\kappa$, starting from a certain threshold value of $\kappa$, which depends on $c$ and $V_{L}$. These solutions correspond to the two actual bands in the system. Respective values of $\varepsilon$ are then determined by the real part of (24), which constitutes together with (25) the parametric solution of (24):

$$
\frac{c V_{L}\left[1-V_{L} \kappa(2 \ln \kappa \cos \varphi-(2 \varphi-\pi) \sin \varphi)\right]}{\left[1-V_{L} \kappa(2 \ln \kappa \cos \varphi-(2 \varphi-\pi) \sin \varphi)\right]^{2}+\left[V_{L} \kappa(2 \ln \kappa \sin \varphi+(2 \varphi-\pi) \cos \varphi)\right]^{2}}+\kappa \cos \varphi=\varepsilon .
$$

Accordingly, the applicability criterion in the same variables reads

$$
|R(\varepsilon)| \approx\left|\frac{\ln \kappa+1+i\left(\varphi-\frac{\pi}{2}\right)}{\ln \kappa+\left(\varphi-\frac{\pi}{2}\right) \cot \varphi}\right| \leq \frac{1}{2} .
$$

The spectrum rearrangement can be outlined by simple estimations. At a first glance, it may seem that $\varepsilon=\sigma=c V_{L}$ can serve as a solution of (24). In reality, it is easy to ascertain that none of the analytical solutions of (24) is passing through this point. However, there is always an energy in the spectrum, at which $\operatorname{Re}(\varepsilon-\sigma)=0$. Indeed, 
the damping $\operatorname{Im} \sigma$ at this energy is essentially nonzero and is given by (24),

$$
1+\frac{2 c V_{L}^{2} \ln \kappa_{0}}{1+\left(2 V_{L} \kappa_{0} \ln \kappa_{0}\right)^{2}}=0 .
$$

At the low impurity concentration the denominator can be replaced by unity. Thus,

$$
\kappa_{0} \approx \exp \left(-1 /\left(2 c V_{L}^{2}\right)\right) .
$$

Correspondingly, it follows from (25) that $\operatorname{Re} \sigma \approx c V_{L}$. Therefore, the Dirac point gradually shifts away from the resonance with increasing the concentration. Generally speaking, the width of the concentration smearing region around $\varepsilon \approx c V_{L}$, where the applicability criterion (23) is violated, should be also of the order of $\kappa_{0}$.

Both $\left|c V_{L}\right|$ and $\kappa_{0}$ are increasing with the concentration and, when they are straightforwardly extrapolated, simultaneously reach the resonance energy by the order of magnitude. This turns obvious when the expression (29) is recast as $\kappa_{0} \approx-c\left|V_{L}\right|\left(2\left|V_{L}\right| \kappa_{0} \ln \kappa_{0}\right)$. The corresponding impurity concentration $c_{0}=2 \varepsilon_{r}^{2}|\ln | \varepsilon_{r}||$ can be taken as a rough estimate for the critical concentration of the spectrum rearrangement. At the impurity concentration far exceeding this value $\left(c>>c_{0}\right)$, the unity in the denominator of fraction in (28) can be omitted. As a result, the size of the concentration smearing region depends exclusively on the concentration:

$$
\kappa_{0} \sim \sqrt{2 c /|\ln c|}, c>>c_{0} .
$$

The spectrum near the Dirac point can be described in more detail at $c<<c_{0}$. For the small $\kappa$ near the Dirac point (25) is reduced to

$$
\ln \kappa \approx-\frac{1}{2 c V_{L}^{2}}+\left(\frac{\pi}{2}-\varphi\right) \cot \varphi .
$$

Respectively, the equation (26) simplifies to

$$
\varepsilon-c V_{L} \approx \frac{2 c V_{L}^{2}(\pi / 2-\varphi) \kappa}{\sin \varphi} .
$$

Combined, they implicitly yield the energy dependence of the phase,

$$
\varepsilon-c V_{L} \approx \frac{(\pi / 2-\varphi) \mathrm{e}^{(\pi / 2-\varphi) \cot \varphi}}{\sin \varphi} 2 c V_{L}^{2} \mathrm{e}^{-1 /\left(2 c V_{L}^{2}\right)}
$$

Likewise, it follows from the criterion (27) that the width of the concentration smearing region is exponentially small:

$$
\Delta_{R} \approx \mathrm{e}^{-1 /\left(4 c V_{L}^{2}\right)-1} .
$$

By equating two main parameters in (33) with some arbitrary constant $\zeta$,

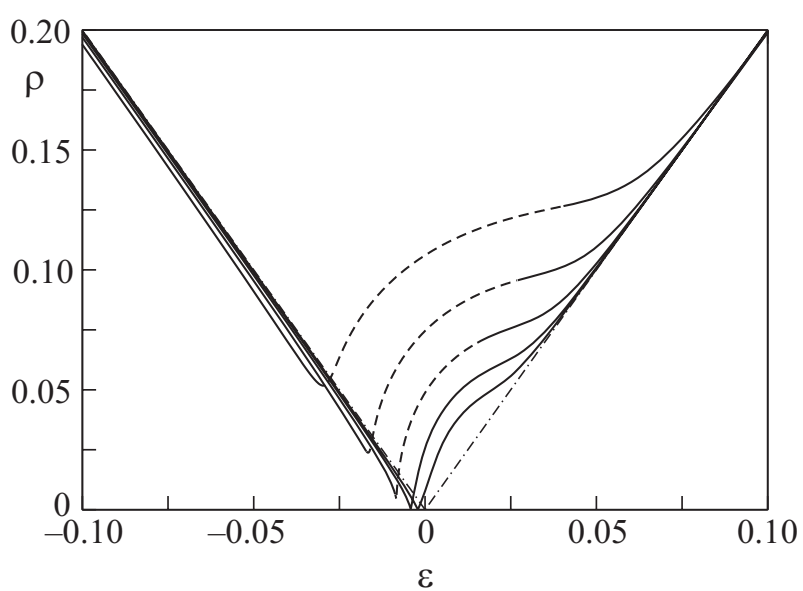

Fig. 1. DOS for $V_{L}=-8$ at $c=2^{n} c_{r}, n=-1 \ldots 3\left(c_{r} \approx 0.0005\right)$.

$$
c V_{L}^{2} \mathrm{e}^{-1 /\left(2 c V_{L}^{2}\right)}=\zeta c\left|V_{L}\right|,
$$

the critical concentration of the spectrum rearrangement can be estimated more precisely:

$$
c_{r}=-\frac{1}{2 V_{L}^{2} \ln \left(\zeta /\left|V_{L}\right|\right)},
$$

which practically coincides with the former rough guess $c_{0}$.

Several examples of results that can be obtained by calculations based on (25)-(27) are presented in Fig. 1. The unperturbed density of states is depicted by the dotdashed line. The fact that the symmetry between the conduction and the valence band is lost at any impurity concentration immediately arrests one's attention. The concentration smearing regions are shown by dashed lines. Initially, only one such region opens up around the Dirac point. In addition, the second one opens near the resonance energy. Both regions merge after a small increase in the concentration. After that, the spectrum outside of the broad concentration smearing region practically does not depend on the magnitude of the impurity perturbation. The fast growth of the concentration smearing region $\sim \sqrt{c}$ was also obtained in [18] in a special case of the infinite in magnitude perturbation $V_{L}$. Despite the single-impurity resonance, the spectrum rearrangement proceeds by the anomalous type in much the same way to the scenario described in [19].

\section{Conclusion}

To summarize, for the moderate impurity perturbation $\left(V_{L}<1\right)$ resonance states does not appear, the spectrum rearrangement does not take place at all, and the width of the concentration smearing region is exponentially small compared to the bandwidth in the whole considered range 
of impurity concentrations. In contrast, for the large impurity perturbation $\left(V_{L}>1\right)$, a resonance state due to a single impurity is manifested in the vicinity of the Dirac point. Still, the width of the concentration smearing region remains exponentially small until the concentration exceeds the critical value $c_{r}$. Then, the width of the concentration smearing region starts to grow fast in magnitude, namely, proportional to $\sqrt{c}$, which means that the spectrum rearrangement of the anomalous type, which is inherent in low-dimensional systems, does take place.

One of the authors (Yu.V.S.) is grateful to B.L. Altshuler and L.A. Pastur for valuable discussions. This work was partially supported by the Scientific Program «Nanostructural Systems, Nanomaterials and Nanotechnologies» of the National Academy of Sciences of Ukraine (grant No. 10/07-N).

1. K. Novoselov, A. Geim, S. Morozov, D. Jiang, Y. Zhang, S. Dubonos, I. Grigorieva, and A. Firsov, Science 306, 666 (2004).

2. M.A. Ivanov and Yu.V. Skripnik, Sov. Phys. Solid State 32, 1722 (1990).

3. M.A. Ivanov and Yu.V. Skripnik, Sov. Phys. Solid State 34, 342 (1992).

4. M.A. Ivanov, V.M. Loktev, and Yu.G. Pogorelov, Phys. Rep. 153, 209 (1987).

5. I.M. Lifshitz, S.A. Gredeskul, and L.A. Pastur, Introduction to the Theory of Disordered Systems, Wiley, N.Y. (1988).
6. A.M. Kosevich, The Crystal Lattice: Phonons, Solitons, Dislocations, Superlattices, WILEY-VCH Verlag GmbH Co. KGaA, Weinheim (2005).

7. S. Zhou, G.-H. Gweon, J. Graf, A. Fedorov, C. Spataru, R. Diehl, Y. Kopelevich, D.-H. Lee, S. Louie, and A. Lanzara, Nature Physics 2, 595 (2006).

8. A. Bostwick, T. Ohta, T. Seyller, K. Horn, and E. Rotenberg, Nature Physics 3, 36 (2007).

9. K. Novoselov, A. Geim, S. Morozov, D. Jiang, M. Katsnelson, I. Grigorieva, S. Dubonos, and A. Firsov, Nature 438, 197 (2005).

10. Y. Zhang, Y.-W. Tan, H. Stormer, and P. Kim, Nature 438, 201 (2005).

11. T. Ando, J. Phys. Soc. Jpn. 74, 777 (2005).

12. I.M. Lifshitz, Adv. Phys. 13, 483 (1964).

13. C. Bena and S.A. Kivelson, Phys. Rev. B72, 125432 (2005).

14. R.J. Elliott, J.A. Krumhansl, and P.L. Leath, Rev. Mod. Phys. 46, 465 (1974).

15. M.A. Ivanov, Sov. Phys. Solid State 12, 1508 (1971).

16. M.A. Ivanov and Yu.V. Skripnik, Fiz. Tverd. Tela (Leningrad) 36, 94 (1994).

17. Yu. Skrypnyk, Phys. Rev. B70, 212201 (2004).

18. V.M. Pereira, F. Guinea, J.M.B. Lopes dos Santos, N.M.R. Peres, and A.H. Castro Neto, Phys. Rev. Lett. 92, 036801 (2006).

19. Yu.V. Skrypnyk and B.I. Min, Progr. Theor. Phys. 108, $1021(2002)$. 